\title{
Long-Circulating Near-Infrared Fluorescence Core-Crosslinked Polymeric Micelles: Synthesis, Characterization, and Dual Nuclear/Optical Imaging
}

\author{
Zhi Yang ${ }^{\dagger} \#$, Shiying Zheng $§$, William J. Harrison $\ddagger$, John Harder $\ddagger$, Xiaoxia Wen ${ }^{\dagger}$, Juri G. \\ Gelovani ${ }^{\dagger}$, Alex Qiao ${ }^{\ddagger}$, and Chun $\mathrm{Li}^{\dagger}$ \\ $\dagger$ Department of Experimental Diagnostic Imaging, The University of Texas M. D. Anderson Cancer Center, \\ Houston, Texas 77030
}

\$Carestream Health, Inc., 1049 Ridge Road West, Rochester, NY 14615

§Eastman Kodak Company Research Labs., 1999 Lake Avenue \#710, Rochester, NY 14650

\begin{abstract}
We report the synthesis of PEG-coated, core-crosslinked polymeric micelles (CCPMs) derived from an amine-terminated amphiphilic block copolymer, poly(PEG-methacrylate)- $b$-poly(triethoxysilyl propylmethacrylate) (PPEGMA- $b$-PESPMA). The block copolymer self-assembled to form micellar nanoparticles, and a Cy-7-like near-infrared fluorescence dye was entrapped in the core bearing reactive ethoxysilane functional groups through a subsequent sol-gel process. The fluorescent signal of CCPMs on the molar basis was 16 -fold brighter than that of Cy7. With an average diameter of 24 $\pm 8.9 \mathrm{~nm}$, CCPMs exhibited prolonged blood half-life $\left(\mathrm{t}_{1 / 2, \alpha}=1.25 \mathrm{hr} ; \mathrm{t}_{1 / 2, \beta}=46.18 \mathrm{hr}\right)$ and moderate uptake by the mononuclear phagocytic system. Significant accumulation of CCPMs in human breast tumor xenografts allowed noninvasive monitoring of the uptake kinetics with both NIRF optical and gamma imaging techniques. Our data suggest that Cy7-entrapped CCPM nanoparticles are suitable for NIRF imaging of solid tumors and have potential applications in the imaging of tumor-associated molecular markers.
\end{abstract}

\section{Keywords}

Near-Infrared Fluorescence; Optical Imaging; Gamma Scintigraphy; Nanoparticles; Polymeric Micelles

\section{Introduction}

Optical imaging is increasingly used to determine protein function and gene expression in live animals. Of the various optical imaging techniques investigated to date, near-infrared (NIR, 700- to 900-nm wavelength) fluorescence (NIRF) imaging is of particular interest for noninvasive in vivo imaging because of the relatively low tissue absorbance and minimal autofluorescence of NIR light. ${ }^{1-3}$ A number of NIRF contrast-enhanced optical imaging probes have been developed and evaluated in small animals. ${ }^{4-10}$ These studies have

\footnotetext{
*Corresponding author: Chun Li, Department of Experimental Diagnostic Imaging—Unit 59, The University of Texas M. D. Anderson Cancer Center, 1515 Holcombe Boulevard, Houston, Texas 77030. Phone: (713) 792-5182. Fax: (713) 794-5456. E-mail address: cli@di.mdacc.tmc.edu.

${ }^{\#}$ On leave from Department of Nuclear Medicine, Peking University School of Oncology \& Beijing Cancer Hospital, Beijing, China 100036
} 
established the use of NIR optical imaging in diagnosis, molecular characterization, and monitoring of treatment response in a variety of disease models. The successful translation of NIRF optical imaging to clinical use will require advances on several fronts, including the development of tomographic optical imaging systems capable of imaging deep organs in vivo, 11,12 the development of endoscopes, laparoscopes, and other intraoperative imaging devices to detect fluorophores at body surfaces, ${ }^{10}$ and particularly, the development and validation of fluorescence-based contrast agents.

Nanoparticles are engineered materials typically smaller than $200 \mathrm{~nm}$, which is small enough to reach almost anywhere in the body. They can be easily derivatized with a variety of targeting ligands and can be loaded with multiple molecules of a contrast agent, providing a huge boost in signal intensity for diverse imaging modalities. ${ }^{13}, 14$ NIRF imaging based on nanoparticulate imaging probes is rapidly emerging as a synergistic enabler in noninvasive cancer detection. Moore et al. ${ }^{15}$ described crosslinked superparamagnetic ion oxide nanoparticles targeted to MUC-1 for dual magnetic resonance imaging (MRI) and NIRF imaging. Several reports have described quantum dots (QDs) composed of a fluorescent core encapsulated within novel polymeric or lipid-based layers for NIRF optical cancer imaging in animals. ${ }^{16-20}$ However, most QDs are made of toxic materials such as cadmium, and it has not yet been established that QDs are sufficiently stable to avoid becoming toxic in the body.

It is well accepted that colloidal particles can preferentially accumulate in tumor after their systemic administration because of the enhanced permeability and retention (EPR) effect, which is characterized by microvascular hyperpermeability to circulating colloidal particles and impaired lymphatic drainage in tumor tissues. ${ }^{21}$ This passive manner of delivery without specific binding to cellular targets (i.e., passive targeting) can be highly effective for watersoluble macromolecules and polymeric micelles. 22,23 It has been recognized, however, that the tumor accumulation of colloidal particles due to the EPR effect can only be successful when the blood circulation time of the particles is prolonged. A number of factors, such as size, size distribution, composition, and surface hydrophilicity, can influence the circulation of nanoparticles in the blood. In particular, surface modification with flexible, hydrophilic poly (ethylene glycol) (PEG) has proven to be effective in preventing the uptake of various polymerbased nanoparticles by the cells of the mononuclear phagocytic system (MPS). 24,25 Here, we report the synthesis of PEG-coated, core-crosslinked polymeric micelles (CCPMs) derived from an amine-terminated amphiphilic block copolymer. We show that these nanoparticles exhibited prolonged blood half-life and enhanced uptake in tumor, suggesting their potential applications in tumor diagnosis and molecular imaging.

\section{Experimental Section}

\section{Materials}

Common reagents were purchased from Sigma-Aldrich (St. Louis, MO) or Acros (Geel, Belgium) and used as received unless otherwise specified. Cy7-like hydrophobic dye 3(triethoxysilyl)propyl-Cy7 was obtained from Eastman Kodak Company (Rochester, NY). pIsothiocyanatobenzyl-diethylenetriaminepentaacetic acid (DTPA-Bz-NCS) was purchased from Macrocyclics (Dallas, TX). Indium-111 chloride $\left({ }^{111} \mathrm{InCl}_{3}\right)$ was obtained from Iso-Tex Diagnostics (Houston, TX).

\section{Analytical Methods}

${ }^{1} \mathrm{H}$ and ${ }^{13} \mathrm{C}$ NMR spectra were recorded on a Varian XL-300 spectrometer (Varian, Inc., Palo Alto, CA) operating at $300 \mathrm{MHz}$ with tetramethyl silane (TMS) as an internal standard. Molecular weights were determined with gel permeation chromatography (GPC) using poly (methyl methacrylate) with narrow molecular weight distribution as standards. The samples 
were separated using two $7.5 \times 300 \mathrm{~mm}$ PLgel mixed-C columns (Polymer Laboratories, Amherst, MA) eluted with 1,1,1,3,3,3-hexafluoroisopropanol containing $0.01 \mathrm{M}$ tetraethyl ammonium nitrate at a flow rate of $1.0 \mathrm{~mL} / \mathrm{min}$. Particle size was measured using dynamic light scattering with a Malvern ZetaSizer Nano-ZS system (Malvern Instruments Ltd, Worcestershire, UK). Zeta potential was determined using a ZetaPlus Analyzer (Brookhaven Instruments Corp., Holtsville, NY).

\section{2-Aminoethyl 2-Bromoisobutyrate}

A solution of $t$-Boc-aminoethyl alcohol (50 g, $0.31 \mathrm{~mol})$ and triethylamine $(34.5 \mathrm{~g}, 0.34 \mathrm{~mol})$ in $300 \mathrm{~mL}$ of methylene chloride was cooled in an ice bath. Into the solution was added 2bromoisobutyryl bromide $(71.3 \mathrm{~g}, 0.31 \mathrm{~mol})$ in $150 \mathrm{~mL}$ of methylene chloride. The reaction was slowly warmed to room temperature and stirred for $4 \mathrm{~h}$. The salt was filtered off and the reaction mixture was extracted sequentially with water and saturated sodium bicarbonate solution. The organic phase was dried over magnesium sulfate. Solvent was evaporated and the crude product was purified by column chromatography using heptane/diethyl ether (80/20, $\mathrm{w} / \mathrm{w}$ ) as an eluent to give $65 \mathrm{~g}$ of $t$-Boc-aminoethyl 2-bromoisobutyrate as a white solid (yield 92\%). ${ }^{1} \mathrm{H}$ NMR $\left(\mathrm{CDCl}_{3}\right) \delta(\mathrm{ppm}): 1.45(\mathrm{~s}, 9 \mathrm{H}), 1.95(\mathrm{~s}, 6 \mathrm{H}), 3.42-3.48(\mathrm{~m}, 2 \mathrm{H}), 4.24(\mathrm{t}, \mathrm{J}=$ $5.25 \mathrm{~Hz}, 2 \mathrm{H})$.

$t$-Boc-aminoethyl 2-bromoisobutyrate $(5.00 \mathrm{~g}, 0.016 \mathrm{~mol})$ was treated with $15 \mathrm{~mL}$ of trifluoroacetic acid under vigorous stirring to remove the $t$-Boc protection group. After the reaction, excess trifluoroacetic acid was removed and the crude product was dried under a vacuum overnight. The crude product was stirred with a mixture of hexane and ethyl acetate and filtered to give $3.18 \mathrm{~g}$ of trifluoroacetic acid salt of 2-aminoethyl 2-bromoisobutyrate as a white crystalline powder (yield 94\%). ${ }^{1} \mathrm{H}$ NMR $\left(\mathrm{CDCl}_{3}\right) \delta(\mathrm{ppm}): 1.93(\mathrm{~s}, 6 \mathrm{H}), 3.35$ (s, br, 2 $\mathrm{H}), 4.46(\mathrm{t}, \mathrm{J}=5.1 \mathrm{~Hz}, 2 \mathrm{H}), 8.10(\mathrm{~s}, \mathrm{br}, 3 \mathrm{H}) ;{ }^{13} \mathrm{C} \mathrm{NMR}\left(\mathrm{CDCl}_{3}\right) \delta(\mathrm{ppm}): 30.24,38.76,55.52$, $61.99,161.49,161.96,162.43,162.90,171.78$.

\section{Block Copolymer PPEGMA- $b$-PESPMA $(x=31, y=46)$}

A 50-mL 3-neck flask was equipped with an additional funnel, a stopper, and a septum. Trifluoroacetic acid salt of 2-aminoethyl 2-bromoisobutyrate $(0.106 \mathrm{~g}, 0.33 \mathrm{mmol})$ and bipyridine ( $174 \mathrm{mg}, 1.11 \mathrm{mmol}$ ) were dissolved in $2.4 \mathrm{~mL}$ of dry methanol in the flask, and methoxy-PEG methacrylate (PEG-MA, MW 475) (4.8 g, $0.01 \mathrm{~mol})$ was added.

Triethoxysilylpropyl methacrylate (ESP-MA) (vacuum distilled from $\left.\mathrm{CaH}_{2}\right)(4.5 \mathrm{~mL}, 4.41 \mathrm{~g}$, $0.015 \mathrm{~mol}$ ) was placed in the additional funnel with $2.0 \mathrm{~mL}$ of dry methanol. Both mixtures in the flask and additional funnel were degassed by bubbling nitrogen for 15 minutes. Then $\mathrm{CuBr}$ ( $80 \mathrm{mg}, 0.56 \mathrm{mmol}$ ) was added quickly to the flask, and the solution turned dark brown. The flask was heated in an oil bath at $50^{\circ} \mathrm{C}$ for 30 minutes and ESP-MA solution was added quickly to the flask. The polymerization was continued overnight at $50^{\circ} \mathrm{C}$. The polymerization mixture was then diluted with dry THF, passed through a pad of celite and basic aluminum twice, and concentrated to yield a clear, viscous semi-solid. ${ }^{1} \mathrm{H}$ NMR indicated the ratio of $\mathrm{x} / \mathrm{y}$ to be close to $1 / 1.5 .{ }^{1} \mathrm{H}$ NMR (300 MHz, $\left.\mathrm{CDCl}_{3}\right) \delta(\mathrm{ppm})$ : 0.55 (s, br), 0.77 (s, br), 0.95 (s, br), 1.18 (t, $\mathrm{CH}_{3}$ from $\left.\mathrm{Si}\left(\mathrm{OCH}_{2} \mathrm{CH}_{3}\right)\right), 1.64$ (s, br), 1.96 (s, br), 3.31 (s, OMe), 3.58 (s, br, $\mathrm{PEG}$ ), 3.75 (m, $\mathrm{CH}_{2}$ from $\left.\mathrm{Si}\left(\mathrm{OCH}_{2} \mathrm{CH}_{3}\right)\right), 4.01$ (s, br).

\section{NIRF CCPM Nanoparticles}

The overall process consisted of two steps. Polymeric micelles were first formed upon the gradual addition of distilled water into a THF solution of the block copolymer and Cy7-like dye 3-(triethoxysilyl)propyl-Cy7. This was followed by the addition of acetic acid to induce a crosslinking reaction among ethoxysilyl groups. Briefly, block copolymer PPEGMA- $b$ PESPMA (100 mg) and 3-(triethoxysilyl)propyl-Cy7 $(0.4 \mathrm{mg})$ were dissolved in $10 \mathrm{~mL}$ of THF. Ten $\mathrm{mL}$ of distilled water was then added slowly to the THF solution. The mixture was 
stirred at room temperature in the dark for $8 \mathrm{hr}, 0.05 \mathrm{~mL}$ acetic acid was added, and the mixture was stirred again at room temperature overnight. For purification, the nanoparticle-containing solution was dialyzed against distilled water for $24 \mathrm{hr}$ (MW cutoff, 3000). The solution was then filtered sequentially through $0.7,0.45,0.2$, and $0.1 \mu \mathrm{m}$ filters. The solution could be concentrated to the desired concentration by centrifugal filtration using a membrane with a molecular weight cutoff of 30,000.

\section{DTPA-Conjugated CCPM Nanoparticle}

An aqueous solution of CCPM nanoparticles $(20 \mathrm{~mL}, 9.3 \mathrm{mg}$ dry weight $/ \mathrm{mL}$ water) was placed in an amber vial. The $\mathrm{pH}$ of the solution was adjusted to 7.5 using $2 \%$ sodium bicarbonate solution. DTPA-Bz-NCS $(0.6 \mathrm{mg}, 1 \mathrm{mg} / \mathrm{mL}$ water) was added to the vial. The reaction was stirred under nitrogen overnight. Unreacted DTPA-Bz-NCS was removed by centrifugal filtration using a membrane with a molecular weight cutoff of 30,000. Approximately $70 \%$ of the DTPA used was attached to the particles.

\section{Estimate of Number of DTPA per CCPM Particle}

DTPA loading per particle was estimated on the basis of the amount of total DTPA-Bz-NCS added initially and unreacted DTPA-Bz-NCS after the reaction. The unreacted DTPA-Bz-NCS was quantified by capillary electrophoresis. Capillary electrophoresis was performed on a Beckman Coulter (Fullerton, CA) P/ACE MDQ system. Data acquisition was made through a Dionex Universal Chromatography Interface, UCI-100, employing Dionex Chromeleon software, version 6.6 (Dionex Corporation, Sunnyvale, CA). The system was eluted with 10.0 $\mathrm{mM}$ acetate buffer ( $\mathrm{pH} 4.75$ ) containing $10 \mathrm{mM}$ copper sulfate and $3.0 \mathrm{mM}$ myristyltrimethylammonium bromide at $25.0^{\circ} \mathrm{C}$. The DTPA-Cu complex formed permitted direct UV detection at $254 \mathrm{~nm}$. Samples were diluted to a final volume of $2.0 \mathrm{~mL}$, providing a working concentration in the 0.5 to $5 \mathrm{mg} / \mathrm{L}$ range. DTPA was added as an internal standard at $5.0 \mathrm{mg} / \mathrm{L}$. Both the hydrolyzed product DTPA-Bz- $-\mathrm{NH}_{2}$ and the unhydrolyzed product DTPA-Bz-NCS were quantified, and the sum of both products was used in the calculation. Approximately $70 \%$ of the DTPA used was attached to the particles. This number, along with the number of amino end groups per particle (180 amines) and the feed molar ratio between DTPA-Bz-NCS and amino groups (15\%), was used to calculate the number of DTPA attached to each particle: $\#$ DTPA/particle $=180 \times 15 \% \times 70 \%=19$. The number of amino end groups per particle was calculated according to weight per particle/molecular weight per copolymer chain $\times 6.02 \times 10^{23}$, in which the weight per particle was calculated based on the volume per particles $\left(4 \pi \mathrm{r}^{3} / 3\right)$ and particle density of $1.0 \mathrm{~g} / \mathrm{cm}^{3}$.

\section{Radiolabeling}

Aliquots of DTPA-CCPM in $0.1 \mathrm{M}$ sodium acetate solution ( $\mathrm{pH}$ 5.2) was mixed with an aqueous solution of ${ }^{111} \mathrm{InCl}_{3}$ for $30 \mathrm{~min}$. Radiolabeled nanoparticles were analyzed using an instant thin layer chromatography (ITLC) system. The ITLC strips were developed with phosphate buffered solution ( $\mathrm{pH}$ 7.4) containing $4 \mathrm{mM}$ EDTA and quantified using a Bioscan IAR-2000 TLC Imaging Scanner (Washington, DC). Free ${ }^{111} \mathrm{In}^{3+}$ moved to the solvent front $(\mathrm{Rf}=0.9)$, and the nanoparticles remained at the original spot $(\mathrm{Rf}=0.0)$. The labeling efficiency for CCPMs was $>98 \%$. The specific activity was $1.18 \mathrm{mCi} / \mathrm{nmol}$.

\section{Cell Line}

Human breast MDA-MB468 cell line was obtained from American Type Cell Culture (Rockville, MD). Cells were maintained at $37^{\circ} \mathrm{C}$ in a humidified atmosphere containing $5 \%$ $\mathrm{CO}_{2}$ in Dulbecco's modified Eagle's medium and nutrient mixture F-12 Ham (DMEM/F12) containing $10 \%$ fetal bovine serum (GIBCO, Grand Island, NY). 


\section{Pharmacokinetic Studies}

All animal studies were carried out in the Small Animal Imaging Facility at The University of Texas M. D. Anderson Cancer Center in accordance with institutional guidelines. Healthy female BALB/c mice (22-25 g; Charles River Laboratories, Wilmington, MA) $(\mathrm{n}=8)$ were injected intravenously at a dose of $5 \times 10^{13}$ particles per mouse $(60 \mu \mathrm{Ci} /$ mouse $)$. At predetermined intervals, blood samples $(10 \mu \mathrm{L})$ were taken from the tail vein, and the radioactivity of each sample was measured with a Cobra Autogamma counter (Packard, Downers Grove, IL). The blood pharmacokinetic parameters for the radiotracer were analyzed using a two-compartmental model and WinNonlin ${ }^{\mathrm{TM}}$ 5.0.1 software (Pharsight Corp., Palo Alto, CA).

\section{Biodistribution Studies}

Solid human mammary MDA-MB468 tumors $\left(1 \times 10^{6}\right.$ viable tumor cells suspended in PBS $)$ were inoculated subcutaneously in the right forelegs of nude mice (20-25 g; Harlan Sprague Dawley, Inc., Indianapolis, IN). When the tumors had grown to 6-8 $\mathrm{mm}$ in average diameter, the mice were randomly allocated into groups, with each group consisting of 3 mice. Mice in each group were injected intravenously with ${ }^{111}$ In-labeled CCPMs $\left(1.3 \times 10^{14}\right.$ particles/mouse, $150 \mu \mathrm{Ci} /$ mouse) and killed at $48 \mathrm{hr}$ and $120 \mathrm{hr}$ after injection ( $\mathrm{n}=3$ for each time point). Blood, heart, liver, spleen, kidney, lung, stomach, intestine, muscle, bone, and tumor tissues were removed, weighed, and counted for radioactivity with a gamma counter. Uptake of contrast agent in various tissues was calculated as the percentage of the injected dose per gram of tissue. A biodistribution study was also performed by ex vivo analysis of fluorescence intensities of various tissues resected at $120 \mathrm{hr}$ after nanoparticle injection. Tissues were imaged with a Xenogen IVIS-200 imaging system (Alameda, CA) with ICG filter sets (excitation/emission, $710-760 / 810-875 \mathrm{~nm}$ ). The camera settings included maximum gain, $2 \times 2$ binning, $640 \times 480$ pixel resolution, and an exposure time of $0.8 \mathrm{sec}$. The fluorescence intensities of the resected tissues were measured and the data were expressed as flux (photons/s) per gram of tissue.

\section{Imaging Studies}

Nude mice bearing subcutaneous MDA-MB468 tumors were injected intravenously with ${ }^{111}$ In-labeled CCPM nanoparticles at a dose of $1.3 \times 10^{14}$ particles/mouse $(150 \mu \mathrm{Ci} /$ mouse). For both nuclear and optical imaging, mice were placed in the anterior position. Prior to imaging, mice were anesthetized with $2 \%$ isoflurane gas (Iso-Thesia, Rockville, NY) in oxygen. During imaging, mice were maintained in an anesthetized state with $0.5 \%-1.5 \%$ isoflurane. Gamma images were acquired using an M-Cam camera equipped with a mediumenergy collimator and ICON software (Siemens, Hoffman Estates, IL). Images were acquired with the following parameters: martix: $512 \times 512$ pixels; zoom: 3.20 ; energy peak: $247 \mathrm{KeV}$ (15\%) and $172 \mathrm{KeV}(15 \%)$; total counts: $800 \mathrm{~K}$ for 5 -min to 48 -hr data points and $500 \mathrm{~K}$ for 120-hr data point.

Whole-body optical and X-ray imaging were performed at various time points up to $120 \mathrm{hr}$ using a Kodak Molecular Imaging System (New Haven, CT) equipped with Cy7 filter sets (excitation/emission, $730 / 790 \mathrm{~nm}$ ). The field of view was $12.0 \mathrm{~cm}$ in diameter. The camera was set at $4 \times 4$ binning. The exposure time was adjusted for each imaging session so that images acquired could be presented at the same scale without over-exposure. The exposure time for X-ray imaging was $2 \mathrm{~min}$. NIRF optical images and X-ray images were fused together with Kodak Molecular Imaging Systems software V4.0.4.

\section{Results and Discussion}

The amine-terminated amphiphilic block copolymer poly(PEG-methacrylate)- $b$-poly ( triethoxysilyl propylmethacrylate) (PPEGMA- $b$-PESPMA) was prepared by atom transfer 
radical polymerization (ATRP) using aminoethyl 2-bromoisobutyrate as an initiator and $\mathrm{CuBr}$ as a catalyst (Fig. 1). ${ }^{26}$ ATRP is a versatile living polymerization methodology that can tolerate a wide variety of monomers and produce polymers with well-controlled molecular weight and architecture. 27 The polymerization was conducted in methanol at $50^{\circ} \mathrm{C}$ via sequential monomer addition. The ${ }^{1} \mathrm{H}$ NMR spectrum of the raw product reveals the presence of signals from both PPEGMA and PESPMA blocks and the absence of vinyl peaks from the monomers, indicating a complete polymerization reaction (Figure S1 in the Supporting Information). The resulting polymer was purified by passing it through basic aluminum to remove the catalyst and concentrated to a viscous semi-solid. The mole ratio of repeating units between the hydrophilic PPEGMA block and hydrophobic PESPMA block (x/y) was 1/1.5, as determined by ${ }^{1} \mathrm{H}$ NMR analysis, which is consistent with the monomer feed ratio of $x / y=31 / 46$. The molecular weight of the copolymer was controlled by the feed ratio between the monomer and initiator. The theoretical molecular weight of PPEGMA- $b$-PESPMA calculated from 100\% monomer conversion was 28,084 . This is in accordance with molecular weight measured by gel permeation chromatography (GPC). Using linear poly(methacrylic acid) as calibration standards, GPC measurement yielded a number-average molecular weight $\left(\mathrm{M}_{\mathrm{n}}\right)$ of 28,600 and a polydispersity value $\left(\mathrm{M}_{\mathrm{w}} / \mathrm{M}_{\mathrm{n}}\right)$ of 1.3 .

Amine-terminated CCPM nanoparticles were prepared from PPEGMA- $b$-PESPMA and NIRF dye 3-(triethoxysilyl)propyl-Cy7, as illustrated in Figure 1. Micelles formed spontaneously when water was added to the tetrahydrofuran (THF) solution of the block copolymer (10 mg/ $\mathrm{mL}$ ) containing the hydrophobic NIRF fluorophore 3-(triethoxysilyl)propyl-Cy7. The micelles underwent a sol-gel process upon the addition of acetic acid, which caused rapid hydrolysis of the ethoxy silane precursors and subsequent crosslink of the PESPMA core with the Cy7-like dye via Si-O bonds. The crosslinked core provided stability to the micellar nanostructure, thus negating the instability produced by the critical micelle concentration. 28 The resulting CCPMs were purified by dialysis and sequential filtration through $0.7,0.45,0.2$, and $0.1 \mu \mathrm{m}$ filters and concentrated via centrifugal filtration through membranes with a molecular weight cutoff of 30,000 .

The terminal amines allow labeling with the gamma emitter indium-111 $\left({ }^{111} \mathrm{In}\right)$ through a metal chelator, diethylentriaminepentaacetic acid (DTPA), to facilitate quantitative pharmacokinetic and biodistribution studies. ${ }^{111}$ In was used because its relatively long physical half-life $\left(\mathrm{t}_{1 / 2}=\right.$ 2.7 days) allows for thorough evaluation of biodistribution for days after nanoparticle injection. Chemical conjugations, whether the introduction of a radiometal chelator or targeting moieties, require the presence of functional groups on the surface of the CCPMs. For this reason, amine was introduced to the terminus of the hydrophilic block of the copolymer PPEGMA- $b$ PESPMA with the anticipation that upon self-assembly, the amino groups would reside on the shells of CCPMs. The CCPMs were treated with isothiocyanate-benzyl-DTPA (DTPA-BzNCS) in aqueous media at $\mathrm{pH} 7.5$, about $70 \%$ of the DTPA-Bz-NCS used in the reaction attached to the CCPMs, indicating that the amines in CCPMs were readily accessible to reagents in the aqueous media (Fig. 2). The degrees of both NIRF dye loading in CCPMs and DTPA attachment to the nanoparticle surface were controlled through stoichiometry and reaction conditions.

The physicochemical properties of CCPM nanoparticles were characterized with regard to composition, size, surface charge, and optical properties. Each CCPM particle contained approximately $21 \mathrm{Cy} 7-$ like dye molecules calculated on the basis of the molar feed ratio between 3-(triethoxysilyl)propyl-Cy7 and the copolymer, assuming that all dyes added in the crosslinking reaction were consumed. This assumption was made on the basis of our observation that no free dyes could be detected in the dialysate solution. DTPA loading per particle was estimated on the basis of the amount of total DTPA-Bz-NCS added initially and unreacted DTPA-Bz-NCS after the reaction, which was quantified by capillary electrophoresis. 
Specifically for the CCPM nanoparticles used in the current study, approximately 19 DTPA molecules were attached to each nanoparticle. The volume average size of DTPA-CCPM was $24 \pm 8.9 \mathrm{~nm}$, determined by dynamic light scattering measurements. These data agreed well with data obtained with transmission electron microscopy (Figure S2 in the Supporting Information). The nanoparticles had weak positive charge with $\zeta$ potential of +1.2 in PBS buffer at $\mathrm{pH}$ 7.5. Because the weight per particle was $7.23 \times 10^{-18} \mathrm{~g}$ and the molecular weight of each block copolymer was 24,000 after crosslinking, it is estimated that each micellar particle was composed of approximately 180 block copolymer chains (Table S1 in the Supporting Information).

The excitation and emission light intensities for the NIRF CCPM nanoparticles peaked at 755 $\mathrm{nm}$ and $781 \mathrm{~nm}$, respectively. In comparison, the excitation and emission intensities for Cy7 dye peaked at $746 \mathrm{~nm}$ and $773 \mathrm{~nm}$, respectively. The quantum yield for Cy7-like dye in CCPMs was 0.081 , compared with a quantum yield of 0.061 for free $\mathrm{Cy} 7$. This increase in the quantum yield for Cy7-like dye in CCPM may be due to the new microenvironment of the dye, e.g., dye immobilized in the core of the particle. The molar extinction coefficient for free $\mathrm{Cy} 7$ and CCPM-trapped Cy7-like dye were $191,130 \mathrm{M}^{-1} \mathrm{~cm}^{-1}$ and $111,100 \mathrm{M}^{-1} \mathrm{~cm}^{-1}$, respectively. The fluorescence brightness of the dye is proportional to the product of the molar extinction coefficient $(\varepsilon)$ and quantum yield (QY) (see Nature Publishing Group website, http://www.nature.com/focus/cellbioimaging/glossary/index.html). Thus, Cy7-like dye in CCPMs was about $77 \%$ brighter than free Cy7 dye in PBS. However, when the nanoparticles were compared with free Cy 7, NIRF CCPM was about 16-fold brighter than Cy7 because each CCPM particle contained 21 Cy7-like dye molecules (Table S2 in the Supporting Information).

Radiolabeling of the nanoparticles was accomplished through incubation of ${ }^{111} \mathrm{InCl}_{3}$ with DTPA-CCPM in sodium acetate buffer at pH 5.5 at room temperature. Under such conditions, a labeling efficiency of greater than $98 \%$ was achieved without further purification. As indicated by instant thin layer chromatography (ITLC), most radioactivity was associated with ${ }^{111}$ In-labeled CCPM nanoparticles $\left(\mathrm{R}_{\mathrm{f}}=0.0\right)$, with no detectable free ${ }^{111} \mathrm{InCl}_{3}\left(\mathrm{R}_{\mathrm{f}}=0.9\right)$ (Figure S3 in the Supporting Information). Figure 3 shows the activity-time profile of ${ }^{111}$ InDTPA-CCPM. The profile fit well into a two-compartment model that can be mathematically described by the equation:

$$
C_{t}(\% \mathrm{ID} / \mathrm{g} \text { blood })=A \mathrm{e}^{-\alpha \mathrm{t}}+B \mathrm{e}^{-\beta \mathrm{t}}
$$

where A and B are the y intercepts of extrapolated lines for the central and tissue compartments, respectively, and $\alpha$ and $\beta$ are first-order rate constants for the central and tissue compartments, respectively. ${ }^{29}$ The mean equation for ${ }^{111}$ In-DTPA-CCPM was $C_{t}=9.83 \mathrm{e}^{-0.67 t}+$ $17.45 \mathrm{e}^{-0.02 \mathrm{t}}$. Following intravenous administration into healthy female BALB/c mice, ${ }^{111} \mathrm{In}$ DTPA-CCPM nanoparticles exhibited blood half-lives of $1.25 \pm 0.66 \mathrm{hr}$ in the distribution phase $\left(\mathrm{t}_{1 / 2, \alpha}\right)$ and $46.18 \pm 6.86 \mathrm{hr}$ in the clearance phase $\left(\mathrm{t}_{1 / 2, \beta}\right)$. CCPM nanoparticles had a large area-under-the-curve (AUC) value of $1184 \% \mathrm{ID} \cdot \mathrm{h} / \mathrm{g}$ blood and a long mean residence time of $65.54 \pm 9.68 \mathrm{hr}$. Prolonged circulation time is usually achieved by using a relatively long PEG chain (MW >2000 Da or $>45 \mathrm{CH}_{2} \mathrm{CH}_{2} \mathrm{O}$ repeating units). ${ }^{25}$ Our results show that increasing the surface PEG chain density through grafting of short oligomeric PEG chains $(9$ $\mathrm{CH}_{2} \mathrm{CH}_{2} \mathrm{O}$ repeating units) to the side chains of a poly(methacrylate) backbone can be equally effective in extending the blood half-life of nanoparticles (other pharmacokinetic parameters are summarized in Table S3 in the Supporting Information).

Biodistribution of CCPM nanoparticles at $48 \mathrm{hr}$ and $120 \mathrm{hr}$ after injection, as determined by ex vivo measurements of radioactivity, are presented in Figure 4A. At $48 \mathrm{hr}$ postinjection, CCPM nanoparticles showed considerably high blood retention, with $8.5 \%$ of the injected dose remaining in the bloodstream. In addition to the blood pool activity, relatively high uptake of nanoparticles in both the liver $(6.3 \% \mathrm{ID} / \mathrm{g})$ and the spleen $(11.3 \% \mathrm{ID} / \mathrm{g})$, compared with that 
among the other major organs, was also observed. In the tumor, ${ }^{111}$ In-DTPA-CCPM had a high uptake value of $5.5 \% \mathrm{ID} / \mathrm{g}$. By $120 \mathrm{hr}$ postinjection, a significant amount of the nanoparticles had been cleared from the organs studied, with the exception of the liver, the spleen, and the tumor (Fig. 4A). In fact, uptake values in the liver and the tumor increased moderately, indicating continuous distribution into these tissues. The tumor-to-blood ratio increased from 0.65 at $48 \mathrm{hr}$ postinjection to 4.44 at $120 \mathrm{hr}$ postinjection. The tumor-to-muscle ratio increased from 9.8 to 28.0 during the same time period.

The biodistribution of CCPM nanoparticles was also determined by ex vivo analysis of the fluorescence signal intensities of resected tissues (Fig. 4B\&C). In accordance with data obtained using the radioactivity count method, the highest activity was found in the liver, the spleen, and the tumor. The ratios of nanoparticle uptake between the tumor and the heart, liver, spleen, kidney, lung, stomach, and muscle were 11.0, 1.02, 1.58, 5.85, 3.25, 4.92, and 25.7, respectively, at $120 \mathrm{hr}$ postinjection, as determined by measurement of the fluorescence intensity. The same ratios were $8.77,0.87,0.75,4.64,4.58,8.37$, and 28.0 , respectively, by the radioactivity count method, representing variations of $25 \%, 11.7 \%, 110 \%, 26 \%,-29 \%$, $-41 \%$, and $-8 \%$, respectively, between the two counting methods. These data suggest that $e x$ vivo measurement of fluorescence intensity may have underestimated the uptake of NIRF nanoparticles in organs with high blood perfusion (i.e., by $11 \%-110 \%$ for the heart, liver, spleen, and kidney). Fluorescence measurement may have also underestimated the uptake in the tumor compared to the radioactivity count method, resulting in a decrease in uptake ratios $(-8 \%$ to $-41 \%)$ between the tumor and the lung, stomach, and muscle. Because measurement of fluorescence intensity is intrinsically less accurate than the radioactivity count method, our data suggest that the attenuation of the fluorescence signal should be corrected in different tissues when estimating the biodistribution of NIRF dyes using the fluorescence method.

Figure 5 shows representative gamma and optical images obtained at various times after injection of ${ }^{111}$ In-DTPA-CCPM nanoparticles at a dose of $1.3 \times 10^{14}$ particles/mouse, corresponding to $150 \mu \mathrm{Ci} /$ mouse and $4.5 \mathrm{nmol}$ fluorophores per mouse. The nuclear images showed a general concordance with the biodistribution data: CCPM nanoparticles exhibited sustained blood pool activity, prominent and persistent accumulation in the liver and the spleen for up to $120 \mathrm{hr}$, and gradual accumulation in the tumor. The cardiac area was clearly seen up to $45 \mathrm{hr}$ postinjection, reflecting a long blood circulation time for CCPMs. No radioactive excretion through the hepatobiliary route was detected. Uptake in the tumor was clearly visualized by $120 \mathrm{hr}$ postinjection with $\gamma$-scintigraphy when the tumor-to-blood ratio increased to greater than 1 (Fig. 5). It was also noted that a small fraction of radioactive species $(<14 \%$ of total injected dose) was excreted into the bladder at 5 min postinjection. ITLC analysis of urine samples collected at $5 \mathrm{~min}$ postinjection revealed that approximately $80 \%$ of the radioactivity in the urine was associated with CCPM nanoparticles (Figure S2 in the Supporting Information). This result suggests that a small fraction of CCPM nanoparticles, possibly the smallest ones, passed through the glomerulus and peritubules of the kidney.

Accumulation of NIRF CCPM nanoparticles in the tumor could be readily visualized at the injected dose (4.5 nmol eq. Cy7/mouse) with optical imaging (Fig. 5). In fact, tumors could be detected at doses up to 100-fold lower than those used in the current study (i.e., $0.045 \mathrm{nmol}$ eq. Cy $7 /$ mouse), indicating that NIRF CCPM nanoparticles displayed a strong and persistent fluorescence signal in vivo. Clearly, $\gamma$-scintigraphy and NIRF optical imaging techniques provided complementary information. While optical imaging offers inherently high sensitivity for superficially localized lesions, $\gamma$-scintigraphy reveals the global distribution of nanoparticles, particularly their uptake in internal organs.

Previous work by Sun et al. ${ }^{30}$ has demonstrated that both the size and core composition of polymeric micelles play an important role in their biodistribution and blood circulation times. 
Interestingly, they also found that surface modification with PEG had no noticeable effect on the biodistribution of the shell-crosslinked polymeric micelles used in their study. The highest reported uptake value in the blood (\%ID/g) at $24 \mathrm{hr}$ postinjection for their series of shellcrosslinked polymeric micelles was $1.56 \pm 0.24 \% .{ }^{30}$ In comparison, CCPM nanoparticles of comparable size $(24 \mathrm{~nm})$ had a blood uptake value of $8.51 \% \mathrm{ID} / \mathrm{g}$ at $48 \mathrm{hr}$ postinjection. In addition to the possible contribution of difference in tumor model used and difference in core composition between CCPM and shell-crosslinked polymeric micelles, crosslinking of the hydrophilic chains in shell-crosslinked polymeric micelles may affect their blood circulation times. The flexibility of the surface PEG chain is thought to contribute to its ability to create an effective steric barrier to disguise PEG-modified nanoparticles and prevent them from being cleared by the MPS. ${ }^{25}$ Shell crosslinking may limit the mobility of the protective layer necessary for preventing the recognition of nanoparticles by the MPS. It may also be possible that the surface charge between CCPMs and those of Sun et al. ${ }^{30}$ is different. Further work is needed to determine which physicochemical characteristics of crosslinked polymeric micelles are important determinants of their pharmacokinetic and biodistribution behaviors. More work is also needed to explain why short PEG chains (9 ethylene oxide repeating units) in CCPMs is sufficient to produce steric hindrance effect to prevent opsonization of nanoparticles as compared to greater than 68 ethylene oxide repeating units (MW > 3000 Da) normally required to achieve such an effect.

\section{Conclusions}

In the current study, we used both ex vivo biodistribution and in vivo dual optical/gamma imaging to evaluate the fate of ${ }^{111} \mathrm{In}$-labeled NIRF CCPM nanoparticles in vivo. We found that CCPM nanoparticles, derived from amphiphilic block copolymer with short PEG chains grafted to the hydrophilic block, were able to remain in circulation for a prolonged time, resulting in effective accumulation in solid tumors. This class of nanoparticles is a promising candidate for dual optical and nuclear imaging applications in tumor detection and molecular imaging. NIRF CCPM offers the opportunity for further surface nano-engineering for targeted molecular imaging applications. Other imaging agents, including those visible on MRI, and therapeutic agents may also be incorporated into the core or shell of the nanoparticles.

\section{Supplementary Material}

Refer to Web version on PubMed Central for supplementary material.

\section{Acknowledgments}

This work is supported by the National Cancer Institute (grant R01 CA119387) and the John S. Dunn Foundation. We thank Professor Mario Ferrari for critical review of the manuscript. We also thank Dawn Chalaire for expert editorial assistance. The animal facility at M. D. Anderson Cancer Center is supported by Cancer Center Support Grant CA16672 awarded by the National Cancer Institute.

\section{References}

1. Ntziachristos V, Bremer C, Weissleder R. Eur Radiol 2003;13:195-208. [PubMed: 12541130]

2. Sokolov K, Follen M, Aaron J, Pavlova I, Malpica A, Lotan R, Richards-Kortum R. Cancer Res 2003;63:1999-2004. [PubMed: 12727808]

3. Gurfinkel M, Ke S, Wen X, Li C, Sevick-Muraca EM. Dis Markers 2003;19:107-121. [PubMed: 15096708]

4. Ballou B, Fisher GW, Waggoner AS, Farkas DL, Reiland JM, Jaffe R, Mujumdar RB, Mujumdar SR, Hakala TR. Cancer Immunol Immunother 1995;41:257-263. [PubMed: 7489569]

5. Folli S, Westermann P, Braichotte D, Pelegrin A, Wagnieres G, van den Bergh H, Mach JP. Cancer Res 1994;54:2643-2649. [PubMed: 8168092] 
6. Achilefu S. Technol Cancer Res Treat 2004;3:393-409. [PubMed: 15270591]

7. Becker A, Hessenius C, Bhargava S, Grotzinger C, Licha K, Schneider-Mergener J, Wiedenmann B, Semmler W. Ann N Y Acad Sci 2000;921:275-278. [PubMed: 11193834]

8. Ke S, Wen X, Gurfinkel M, Charnsangavej C, Wallace S, Sevick-Muraca EM, Li C. Cancer Res 2003;63:7870-7875. [PubMed: 14633715]

9. Wang W, Ke S, Wu Q-P, Charnsangavej C, Gurfinkel M, Gelovani JG, Abbruzzese JL, Sevick-Muraca EM, Li C. Molecular Imaging 2004;3:343-351. [PubMed: 15802051]

10. Weissleder R, Ntziachristos V. Nat Med 2003;9:123-128. [PubMed: 12514725]

11. Godavarty A, Thompson AB, Roy R, Gurfinkel M, Eppstein MJ, Zhang C, Sevick-Muraca EM. Journal of Biomedical Optics 2004;9:488-496. [PubMed: 15189086]

12. Ntziachristos V, Schellenberger EA, Ripoll J, Yessayan D, Graves E, Bogdanov A Jr, Josephson L, Weissleder R. PNAS 2004;101:12294-12299. [PubMed: 15304657]

13. Sullivan DC, Ferrari M. Molecular Imaging 2004;3:364-369. [PubMed: 15802054]

14. Harisinghani MG, Barentsz J, Hahn PF, Deserno WM, Tabatabaei S, van de Kaa CH, de la Rosette J, Weissleder R. N Engl J Med 2003;348:2491-2499. [PubMed: 12815134]

15. Moore A, Medarova Z, Potthast A, Dai G. Cancer Res 2004;64:1821-1827. [PubMed: 14996745]

16. Michalet X, Pinaud FF, Bentolila LA, Tsay JM, Doose S, Li JJ, Sundaresan G, Wu AM, Gambhir SS, Weiss S. Science 2005;307:538-544. [PubMed: 15681376]

17. Smith AM, Gao X, Nie S. Photochem Photobiol 2004;80:377-385. [PubMed: 15623319]

18. Larson DR, Zipfel WR, Williams RM, Clark SW, Bruchez MP, Wise FW, Webb WW. Science 2003;300:1434-1436. [PubMed: 12775841]

19. Gao X, Cui Y, Levenson RM, Chung LW, Nie S. Nat Biotechnol 2004;22:969-976. [PubMed: 15258594]

20. Geng L, Shinohara ET, Kim D, Tan J, Osusky K, Shyr Y, Hallahan DE. Int J Radiat Oncol Biol Phys 2006;64:263-271. [PubMed: 16274936]

21. Matsumura Y, Maeda H. Cancer Res 1986;46:6387-6392. [PubMed: 2946403]

22. Simons M, Bonow RO, Chronos NA, Cohen DJ, Giordano FJ, Hammond HK, Laham RJ, Li W, Pike M, Sellke FW, Stegmann TJ, Udelson JE, Rosengart TK. Circulation 2000;102:E73-E86. [PubMed: 10982554]

23. Kataoka K, Harada A, Nagasaki Y. Adv Drug Deliv Rev 2001;47:113-131. [PubMed: 11251249]

24. Gref R, Minamitake Y, Peracchia MT, Trubetskoy V, Torchilin V, Langer R. Science 1994;263:16001603. [PubMed: 8128245]

25. Owens DE 3rd, Peppas NA. Int J Pharm 2006;307:93-102. [PubMed: 16303268]

26. Pietras K, Rubin K, Sjoblom T, Buchdunger E, Sjoquist M, Heldin CH, Ostman A. Cancer Res 2002;62:5476-5484. [PubMed: 12359756]

27. Coessens V, Pintauer T, Matyjazsewski K. Prog. Polym. Sci 2001;26:337-377.

28. Thurmond, Kn; Remsen, E.; Kowalewski, T.; Wooley, K. Nucleic Acids Res 1999;27:2966-2971. [PubMed: 10390540]

29. Wen X, Wu QP, Ke S, Wallace S, Charnsangavej C, Huang P, Liang D, Chow D, Li C. Cancer Biother Radiopharm 2003;18:819-827. [PubMed: 14629830]

30. Sun X, Rossin R, Turner JL, Becker ML, Joralemon MJ, Welch MJ, Wooley KL. Biomacromolecules 2005;6:2541-2554. [PubMed: 16153091] 


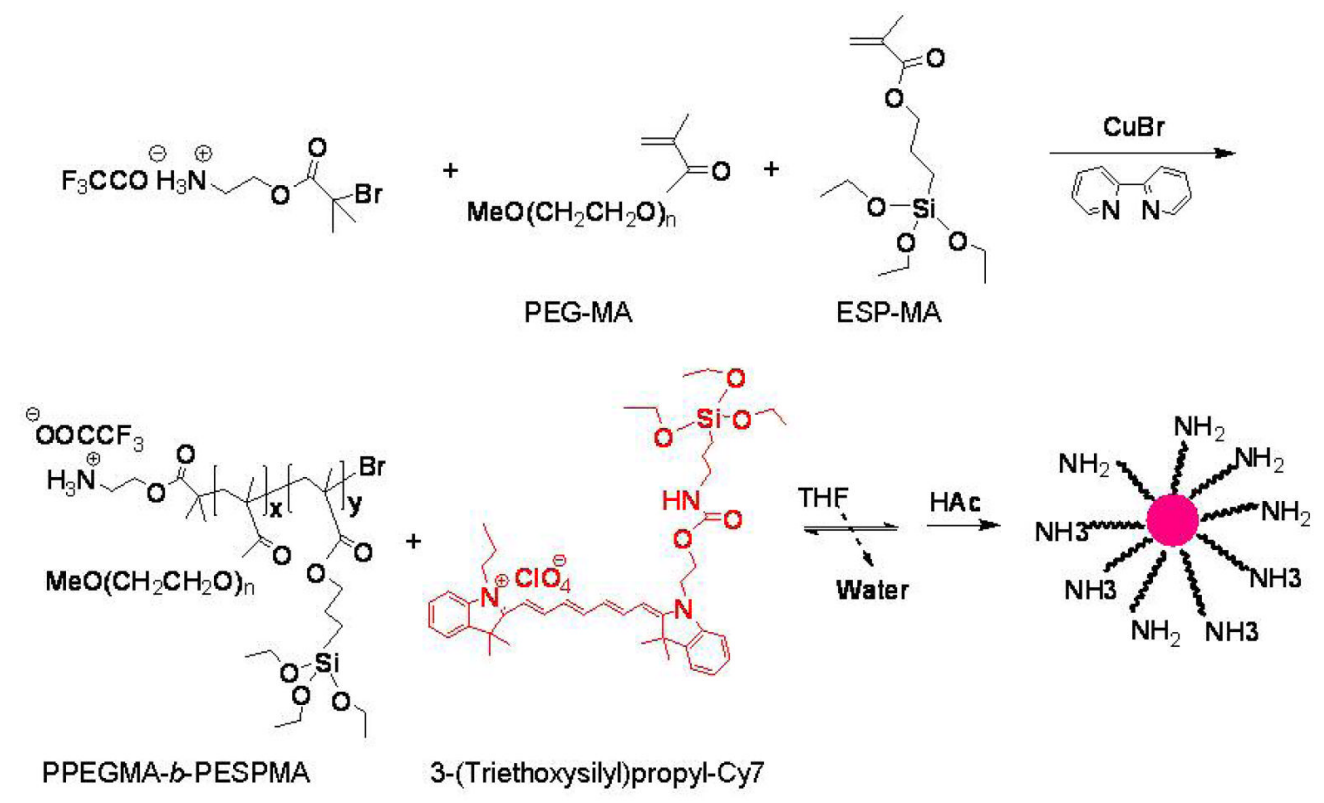

Fig. 1.

Synthesis of PPEGMA- $b$-PESPMA block copolymer and schematic illustration of the preparation of amine-terminated, core-crosslinked polymeric micelles containing NIRF dyes. 


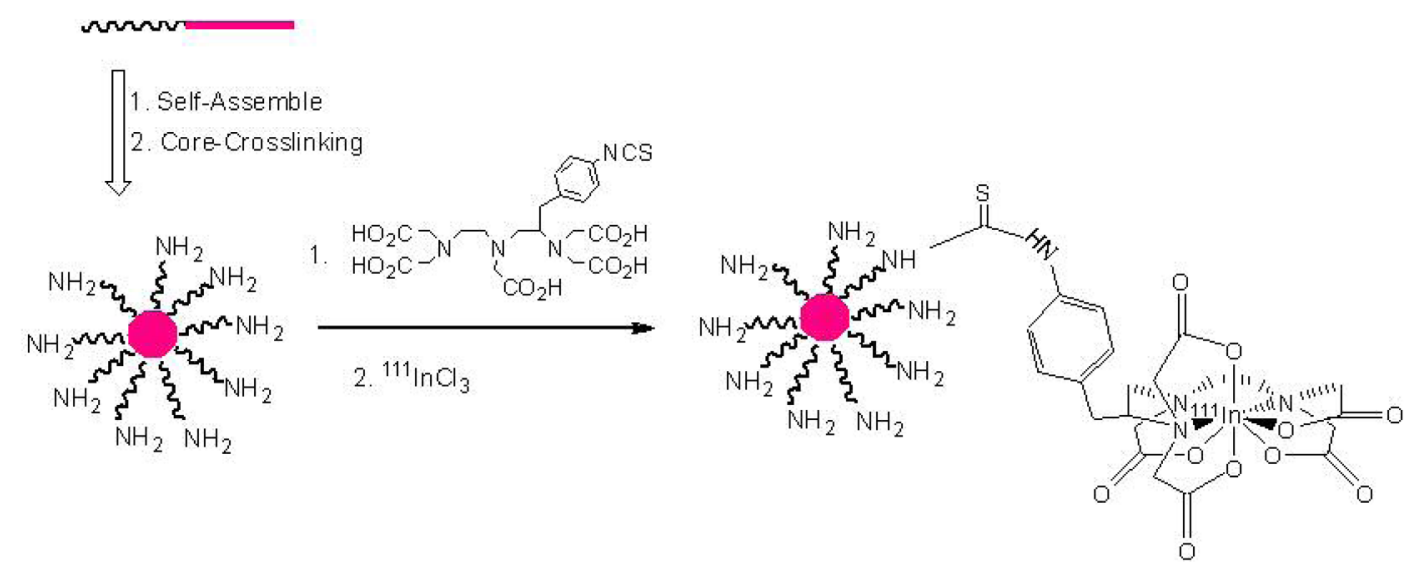

Fig. 2.

Conjugation of radiometal chelator DTPA to the NIRF nanoparticles and labeling with ${ }^{111}$ In. 


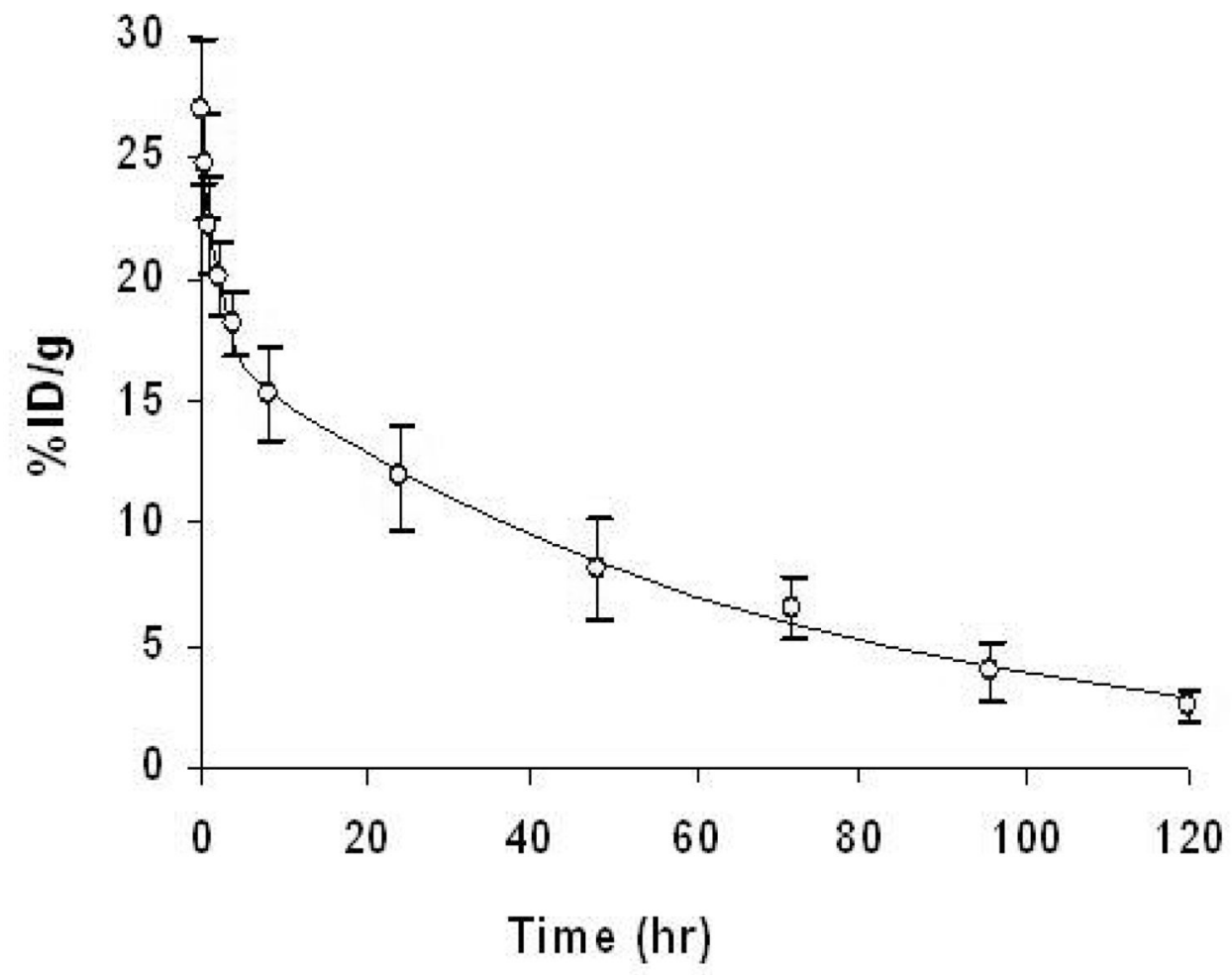

Fig. 3.

Blood activity-time profile of ${ }^{111}$ In-DTPA-CCPM. The open circles represent the mean radioactivity expressed as a percentage of the injected dose per gram of blood from 8 mice. The solid line is a curve fitted to a two-compartment model consisting of a central compartment and a peripheral compartment. Data are expressed as means \pm standard deviation. 

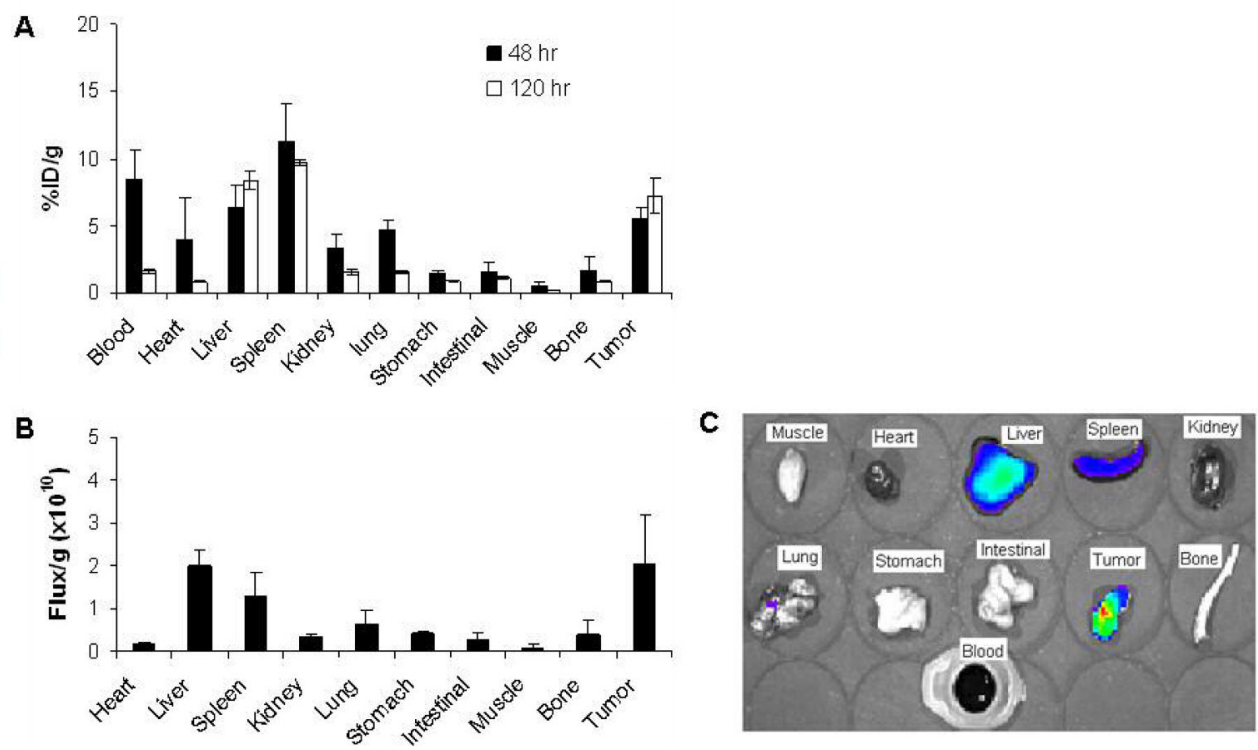

Fig. 4.

Biodistribution of ${ }^{111}$ In-DTPA-CCPM in nude mice bearing subcutaneous MDA-MB-468 tumors. A: Data were obtained using the radioactivity count method, plotted as \% injected dose per gram of tissue (\%ID/g). B: Biodistribution at $120 \mathrm{hr}$ after i.v. injection obtained using the fluorescence intensity measurement method, plotted as photon flux per gram of tissue. All data are expressed as mean \pm standard deviation $(n=3)$. C: Representative NIRF images of various tissues resected from a mouse at the end of the imaging sessions (120 $\mathrm{h}$ after injection). 
Gamma

Scintigraphy
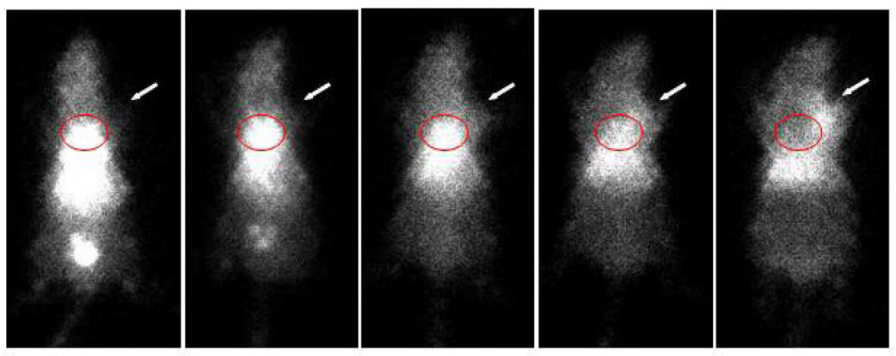

\section{Optical}
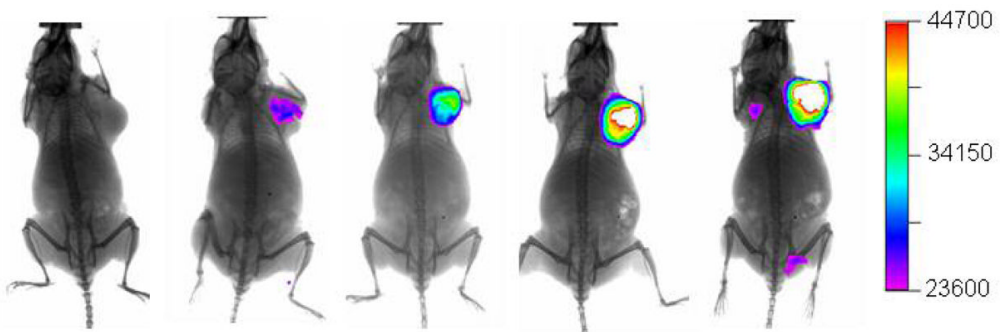

$5 \mathrm{~min}$

$2 \mathrm{~h}$

$20 \mathrm{~h}$

$45 \mathrm{~h}$

$120 \mathrm{~h}$

Fig. 5.

Representative $\gamma$-scintigraphy and NIRF optical images of mice at various times after i.v. injection of ${ }^{111}$ In-DTPA-CCPM. MDA-MB-468 breast tumors (arrows) were inoculated subcutaneously on front legs. The scale bar shows signal intensity in arbitrary units. The radioactivity in the cardiac area is circled in red. 